\title{
Air Quality Monitoring with SensorMap
}

\author{
Péter Völgyesi, András Nádas, Xenofon Koutsoukos, Ákos Lédeczi \\ Institute for Software Integrated Systems \\ Vanderbilt University \\ Nashville, TN, 37235, USA \\ \{peter.volgyesi,andras.nadas, xenofon.koutsoukos,akos.ledeczi\}@vanderbilt.edu
}

\begin{abstract}
The Mobile Air Quality Monitoring Network (MAQUMON) is presented. The system consists of a number of car-mounted sensor nodes measuring different pollutants in the air. The data points are tagged with location and time utilizing an on-board GPS. Periodically, the measurements are uploaded to a server, processed and then published on the SensorMap portal. Given a sufficient number of nodes and diverse mobility patterns, a detailed picture of the air quality in a large area will be obtained at a low cost.
\end{abstract}

\section{Introduction}

Air pollution is one of the most important factors affecting the quality of life and the health of the increasingly urban population of industrial societies. In the U.S., all major cities have networks of monitoring stations providing continuous measurements of the most important pollutants. However, the number of these stations is usually very small. In the Nashville metropolitan area, for example, there are ten such stations sparsely covering the central area of Davidson county [1]. The situation is not much different in others cities. Air pollution is highly locationdependent, for example, the vicinity of traffic chokepoints or certain industrial installations has much worse air quality than average. Furthermore, currently the data of the different pollutants measured at the different stations in the city are aggregated to a single number, the air quality index (AQI), that is published once a day on a website. In other words, there is not enough data gathered to evaluate air quality in a given neighborhood and the publicly available information is even more deficient.

In contrast to monitoring methods using stationary stations, a detailed picture based on real-time data from mobile sensors for the entire populated area would offer major benefits to air quality control. We are building a prototype Mobile Air Quality Monitoring
Network (MAQUMON) comprised of sensor nodes mounted on cars. A sensor node consists of a microcontroller, an on-board GPS unit and set of gas sensors measuring ozone, $\mathrm{CO}$, and $\mathrm{NO} 2$ concentrations. The node is Bluetooth enabled, so it can connect to a PDA or laptop to upload the measurements.

When the car is in motion, the device samples the sensors every minute and store the results tagged with a location and time stamp. When the car is parked, the samples are only taken a few times an hour. When a car is within the coverage area of an available WiFi hotspot, all data are uploaded to our server, processed and published on the SensorMap portal. Given a sufficient number of nodes and diverse mobility patterns, a detailed picture of the air quality in a large area will be obtained at a low cost.

\section{Hardware Platform}

The sensor platform supports autonomous data collection, storage and off-line data retrieval or the streaming of live sensor readings. An integrated Bluetooth module provides a wireless interface for laptop computers or PDAs.

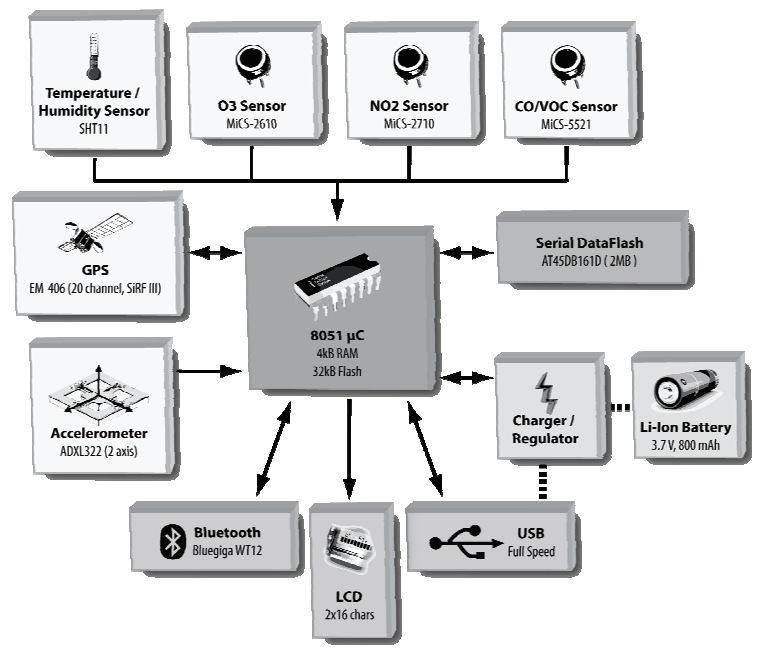

This work has been supported by a gift from Microsoft Research. 
Figure 1. Architecture of the sensor node

Alternatively, the system can be accessed through a USB cable. This wired interface can also provide power to the board both for on-line operation and for charging the integrated Li-ion battery. The battery life of the device is limited to a few hours (in active data acquisition mode), but in the car mounted scenario, it can be constantly powered from the cigarette lighter. Furthermore, a 2-axis MEMS accelerometer is used to detect if the system is in motion and can automatically turn off all the power hungry components (GPS, Bluetooth, gas sensors) if not in use. Location and time information is provided by an on-board 20 channel SiRF-III-based GPS module at $1 \mathrm{~Hz}$ sampling rate. Gas concentration levels are measured by three analog sensors: $\mathrm{O} 3, \mathrm{NO} 2$ and $\mathrm{CO} / \mathrm{VOC}$. These readings along with temperature and relative humidity data and GPS information are stored in a serial flash device $2 \mathrm{MB}$. A $2 \times 16$ character LCD panel provides immediate visual feedback about the status of the system (connected interfaces, GPS lock, time, motion detection, sensor readings). The Intel 8051-based microcontroller controls every aspect of the system from battery charging to analog/digital conversions and the USB protocol.

A photograph of the prototype board in its enclosure is shown below.

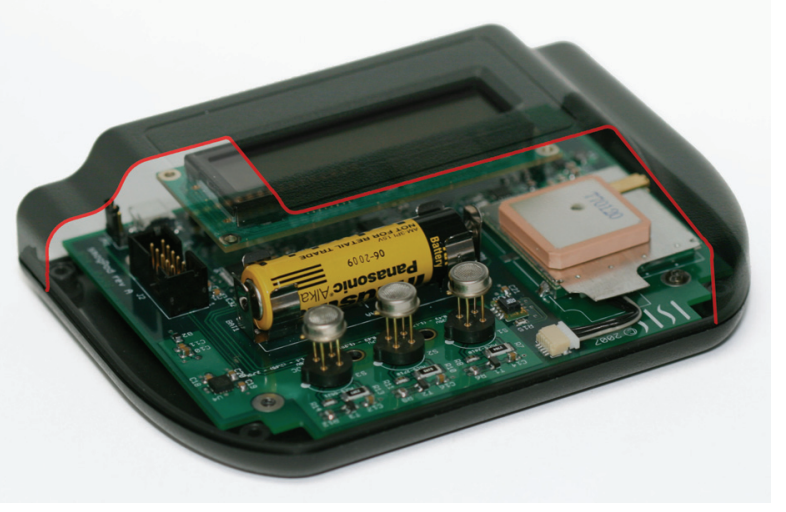

Figure 2. Sensor node prototype

\section{Demonstration}

The demonstration will have two main parts. First, the prototype hardware platform will be shown and its operation demonstrated. While we do not plan to release any of the gases the system can measure in the confined area, the presence of at least one gas can be demonstrated. The breath of humans contains different amounts of $\mathrm{CO}$ depending on the lung function of the individual. For example, smokers have typically higher amounts of CO. We will demonstrate our simple Smoke ${ }^{\circledR}$ Detector application that detects when somebody breathes on the unit using the temperature and humidity sensors and tell whether the person is a smoker or not. The result is displayed on the integrated LCD display of the unit.

The second part of the demonstrations will illustrate the integration with the MSR SensorMap portal [2]. Since gathering data indoors in a small area would be insufficient for this purpose, we will use prerecorded data. Visualizing air quality presents two challenges. First, the sensors are mobile, so they cannot be associated with a single geographic location. Second, it is not data associated with a single sensor that is interesting, but the overall air quality of an area determined by a set of sensors measuring pollutants for a certain amount of time.

Sensor mobility is handled using the SensorMap mobile proxy feature. Overall air quality will be displayed in the form of contour maps utilizing image overlays. The time series data for a given sensor and/or a given geographic location will be also available. The figure below illustrates the visualization techniques employed by the MAQUMON system.

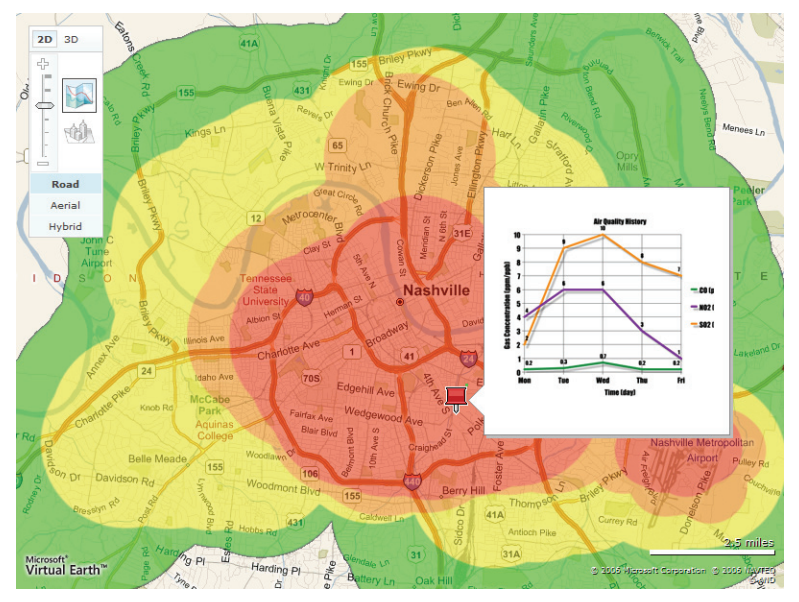

Figure 3. Visualization in SensorMap

\section{References}

[1] Annual Report, Metro Public Health Department of Nashville/Davidson County Pollution Control Division, 2005 http://healthweb.nashville.org/env/aqi/psipoll.asp

[2] Microsoft Research SensorMap Portal, http://atom.research.microsoft.com/sensormap/ 